\title{
Reconstruction of missing boundary conditions from partially overspecified data : the Stokes system
}

\author{
Amel BEN ABDA, Faten KHAYAT \\ LAMSIN-ENIT \\ BP 37, 1002 Tunis le Belvédère, \\ Tunisia. \\ amel.benabda@enit.rnu.tn, faten.khayat@gmail.com
}

\begin{abstract}
|
RÉSUMÉ. Nous nous intéressons à un problème de cauchy mal posé, celui de la complétion de données frontières pour les équations de Stokes. Nous voulons reconstituer les données manquantes sur une partie non accessible de la frontière du domaine à partir de données peu surdéterminées sur la partie accessible. Nous formulons ce problème inverse sous forme de minimisation d'une fonctionnelle de type énergie. Les conditions d'optimalité du premier ordre sont écrites en termes d'équation d'interface utilisant les opérateurs de Stecklov-Poincaré. Nous donnons des résultats numériques attestant l'efficacité de la méthode.
\end{abstract}

ABSTRACT. We are interested in this paper with the ill-posed Cauchy-Stokes problem. We consider a data completion problem in which we aim recovering lacking data on some part of a domain boundary, from the knowledge of partially overspecified data on the other part. The inverse problem is formulated as an optimization one using an energy-like misfit functional. We give the first order optimality condition in terms of an interfacial operator. Displayed numerical results highlight its accuracy.

MOTS-CLÉS : Conditions aux limites défectueuses, Problème de Cauchy-Stokes, Complétion de données, Contrainte de cisaillement, Coefficient de Robin, Stent cérébral.

KEYWORDS : Defective boundary condition, Cauchy-Stokes problem, Data completion, Shear stress, Robin coefficient, Cerebral stent.

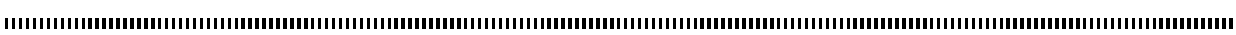




\section{Introduction}

We are interested in this work with the Cauchy-Stokes problem that consists in solving Stokes system on a domain from overspecified boundary data. In the literature, this situation is referred to as the boundary data completion problem.

The most common task in such inverse problem consists in recovering the missing boundary conditions on the inaccessible part of the boundary of known Cauchy data on the accessible part.

There is a huge literature dealing with the scalar case (namely the Laplace operator, see [8] and the references therein) whereas the PDE's systems are less treated. Up to our knowledge, there are few papers dealing with the Cauchy-Stokes system (see [1],[7] and the references therein). In [6, 2], the authors deal with the inverse problem of detecting an immersed body in a fluid. In [10], the authors were concerned with identifying a Robin coefficient on a non accessible part of the boundary from available data on the other part. While in [16], the inverse problem of viscosity's identification was treated.

However, in many engineering applications, and particularly in bioengineering ones, these data are often not complete, i.e. the available data often refers to the velocity field and only one component of the normal stress.

Such kind of systems naturally appears in the modelling of biological problems, one can think of blood flow in cardiovascular system or airflow in the lungs. Such situations may also occur in many other physical studies such as coating flows [12], flows in semiconductor melts [13] or problems related to Newtonian fluid flows at solid interfaces [21]. Our focus in this work is on the reconstruction of the velocity and the pressure.

The Cauchy problem has been known since Hadamard to be severely ill posed. However, we deal here with partially overspecified boundary conditions, namely only one component of the stress tensor is given on the accessible part of the boundary. This seems to be delicate since no unique continuation result is available in this case.

We would like to refer to a new work in this area [17] where these kind of problems has been addressed for the bilaplacian operator in 2D situations.

In this paper, we propose to perform a method based on minimization of an energy-like functional introduced in [7, 3], to the case of partially overdetermined data. This paper is organized in 8 sections. In Section 2, we introduce the partially overdetermined Cauchy problem and report some useful theorical results. Section 3 is devoted to the solvability issues related to the forward problem as well as the inverse one. In Section 4, we formulate the partially overdetermined Cauchy problem as a data completion one and introduce the related minimization problem. For the Neumann-Dirichlet approach, we prove the uniqueness of the minimizer. Using Steklov-Poincaré operators, first-order optimality conditions are obtained in terms of an interfacial transmission problem. The numerical procedure for solving the partially overdetermined Cauchy-Stokes problem is described in Section 5. In Section 6, we present various examples that illustrate the efficiency of the proposed 
method. Then we apply our study to identify a Robin coefficient of the Stokes system : it's the Section 7. Section 8 is devoted to concluding remarks.

\section{Problem setting}

We denote by $H^{1}(\Omega)^{d}=\left\{\boldsymbol{u} \in L^{2}(\Omega)^{d}, \partial \boldsymbol{u}_{i} / \partial x_{j} \in L^{2}(\Omega)\right.$ for $\left.i, j=1, \cdots, d\right\}$ the standard first-order $L^{2}$-based Sobolev space equipped with usual first-order Sobolev norm and $L_{0}^{2}(\Omega)=\left\{q \in L^{2}(\Omega): \int_{\Omega} q=0\right\}$. In addition, for any subset $\Gamma$ a connected component of $\partial \Omega$, we will use frequently the fractional Sobolev space $H^{1 / 2}(\Gamma)$, the space of the traces of elements of $H^{1}(\Omega)^{d}$ on $\Gamma$. We will note by $V^{1}(\Gamma)=H_{00}^{1 / 2}(\Gamma)$ the set of all the restrictions to $\Gamma$ of the functions of $H^{1 / 2}(\partial \Omega)$ that vanish on $\partial \Omega \backslash \Gamma$ and by $V^{-1}(\Gamma)$ it's topological dual space. In the entire text $\Omega \subset \mathbb{R}^{d}$, d=2 or 3 , is a lipschitz bounded and connected domain. Assume that $\partial \Omega$ is split into three parts $\Gamma_{c}, \Gamma_{s}$ and $\Gamma_{i}$ of nonvanishing measure such that $\Gamma_{s}$ is closed and $\Gamma_{c} \cap \Gamma_{s} \cap \Gamma_{i}=\emptyset$. Throughout the paper, we adopt the convention that a boldface character denotes a vector or a tensor. For any vector field $\boldsymbol{v}$ on $\partial \Omega$, we shall denote by $\boldsymbol{v}_{\boldsymbol{n}}$ its normal component while we shall denote by $\boldsymbol{v}_{\tau}$ the projection of $\boldsymbol{v}$ on the tangent hyperplane to $\partial \Omega$. In other words $\boldsymbol{v}_{\boldsymbol{n}}=\boldsymbol{v} \cdot \boldsymbol{n}$ and $\boldsymbol{v}_{\tau}=\boldsymbol{v}-\boldsymbol{v}_{\boldsymbol{n}} \boldsymbol{n}$. The partially overdetermined Cauchy problem we are interested in is defined as follows :

Definition 1 (Partially overdetermined Cauchy problem) Assuming that the given data $\Phi \in V^{1}\left(\Gamma_{c}\right)^{2}$ and $T \in V^{-1}\left(\Gamma_{c}\right)$ are compatible, i.e. that this pair is indeed the shear stress and the Dirichlet data of a unique function $\boldsymbol{u}$, the problem is :

Find $(\varphi, t)=(\boldsymbol{u}, \boldsymbol{\sigma}(\boldsymbol{u}) \boldsymbol{n})_{\mid \Gamma_{i}} \in V^{1}\left(\Gamma_{i}\right)^{2} \times V^{-1}\left(\Gamma_{i}\right)^{2}$ such that $(\boldsymbol{u}, p) \in H^{1}(\Omega)^{2} \times L_{0}^{2}(\Omega)$ be the solution of

$$
\begin{cases}-\nu \Delta \boldsymbol{u}+\nabla p=0 & \text { in } \Omega \\ \nabla \cdot \boldsymbol{u}=0 & \text { in } \Omega \\ \boldsymbol{u}=0 & \text { on } \Gamma_{s} \\ \boldsymbol{u}=\Phi,[\boldsymbol{\sigma}(\boldsymbol{u}) \boldsymbol{n}]_{\tau}=T & \text { on } \Gamma_{c}\end{cases}
$$

where $\nu$ is the viscosity of the fluid, $\sigma$ denotes the stress tensor

$$
\boldsymbol{\sigma}(\boldsymbol{u})_{i j}=-p \delta_{i j}+2 \nu \mathbf{D}(\boldsymbol{u})_{i j}, \quad 1 \leq i, j \leq d
$$

where $\mathbf{D}(\boldsymbol{u})$ is the linear strain tensor defined by

$$
\mathbf{D}(\boldsymbol{u})_{i j}=\frac{1}{2}\left(\frac{\partial \boldsymbol{u}_{i}}{\partial x_{j}}+\frac{\partial \boldsymbol{u}_{j}}{\partial x_{i}}\right), \quad 1 \leq i, j \leq d .
$$

Note that $[\boldsymbol{\sigma}(\boldsymbol{u}) \boldsymbol{n}]_{\tau}$ denotes the tangential component of the stress acting on the boundary, that can be expressed by the symmetrized gradient of $\boldsymbol{u}$ such that $[\boldsymbol{\sigma}(\boldsymbol{u}) \boldsymbol{n}]_{\tau}=$ 
$2 \nu[\mathbf{D}(\boldsymbol{u}) \boldsymbol{n}]_{\tau}$. Therefore this condition does not contain the pressure $p$, which is an essential difference from the pure Neumann condition. It can easily be observed that $(\mathbf{D}(\boldsymbol{u}) \boldsymbol{n})$ is in $L^{2}(\Omega)$ and its divergence also is in $L^{2}(\Omega)$. Thus, $\mathbf{D}(\boldsymbol{u})$ has a trace in $V^{-1}(\partial \Omega)$, which is what we can understand by $(\partial \boldsymbol{u} / \partial \boldsymbol{n}) \cdot \tau$.

Since Hadamard, it is well-known that the Cauchy problem is severely ill-posed. Thus problem (1) has no solution unless overspecified data on $\Gamma_{c}$ are compatible, and if a solution exists, it does not depend continuously on the data $(\Phi, T)$. We expect worse behaviour in our case (i.e. Definition 1).

\section{Solvability issues}

The Stokes equations with different boundary conditions in this text will be formulated variationally. Often the Stokes equations are given with Dirichlet boundary conditions, or a combination of a Dirichlet and Neumann conditions. In this section we recall the existence and uniqueness of weak solution for the following Stokes system with friction boundary conditions :

$$
\begin{cases}-\nu \Delta \boldsymbol{u}+\nabla p=0 & \text { in } \Omega \\ \nabla \cdot \boldsymbol{u}=0 & \text { in } \Omega \\ \boldsymbol{u}=0 & \text { on } \Gamma_{s} \\ {[\boldsymbol{\sigma}(\boldsymbol{u}) \boldsymbol{n}]_{\tau}=T} & \text { on } \Gamma_{c} \\ \boldsymbol{u} \cdot \boldsymbol{n}=0 & \text { on } \Gamma_{c} \\ \boldsymbol{u}=\varphi & \text { on } \Gamma_{i}\end{cases}
$$

While the mathematical literature on Dirichlet and Neumann boundary conditions is vast and the well-posedness results are well known, friction boundary conditions have been studied less extensively. We briefly recall the variational formulation of the problem (2) and solvability issues which will be needed in the sequel. For this aim, we need the following lemma that provides a general integration-by-parts formula and where we denote by $A: B$ the tensorial product between $A$ and $B$ defined as follows : $A: B=\sum_{i, j=1}^{d} A_{i j} B_{i j}$

Lemma 2 If $\boldsymbol{u} \in H^{1}(\Omega)^{d}$ such that $\nabla \cdot \boldsymbol{u}=0$, then $\Delta \boldsymbol{u}=2 \nabla \cdot \mathbf{D}(\boldsymbol{u})$ and

$$
-\int_{\Omega} \nu \Delta \boldsymbol{u} \boldsymbol{v}=\int_{\Omega} 2 \nu \mathbf{D}(\boldsymbol{u}): \mathbf{D}(\boldsymbol{v})-\int_{\partial \Omega} 2 \nu \mathbf{D}(\boldsymbol{u}) \boldsymbol{n v}, \quad \forall \boldsymbol{v}, \boldsymbol{u} \in H^{1}(\Omega)^{d} .
$$

The boundary integral term has to be interpreted as a duality product of $\boldsymbol{v} \in V^{1}(\partial \Omega)^{d}$ with the normal derivative $\nu \mathbf{D}(\boldsymbol{u}) \boldsymbol{n} \in V^{-1}(\partial \Omega)^{d}$. Then a mixed formulation of the 
Stokes equations is obtained by multiplying the first equation of (2) by a test function, integrating over $\Omega$, and applying Lemma 2 as well as the divergence theorem,

$$
\int_{\Omega} 2 \nu \mathbf{D}(\boldsymbol{u}): \mathbf{D}(\boldsymbol{v})-\int_{\Omega} p \nabla \cdot \boldsymbol{v}=\int_{\partial \Omega}(2 \nu \mathbf{D}(\boldsymbol{u}) \boldsymbol{n}-p \boldsymbol{n}) \cdot \boldsymbol{v} .
$$

We focus now on the boundary terms of (4). It follows from the following decomposition [4]

$$
\boldsymbol{v}=\boldsymbol{v}_{\boldsymbol{n}} \boldsymbol{n}+\boldsymbol{v}_{\tau} \tau, \quad \text { and } \quad \mathbf{D}(\boldsymbol{u}) \boldsymbol{n}=[\mathbf{D}(\boldsymbol{u}) \boldsymbol{n}]_{\boldsymbol{n}} \boldsymbol{n}+[\mathbf{D}(\boldsymbol{u}) \boldsymbol{n}]_{\tau} \tau
$$

that $\mathbf{D}(\boldsymbol{u}) \boldsymbol{n} \boldsymbol{v}=[\mathbf{D}(\boldsymbol{u}) \boldsymbol{n}]_{\boldsymbol{n}} \boldsymbol{v}_{\boldsymbol{n}}+[\mathbf{D}(\boldsymbol{u}) \boldsymbol{n}]_{\tau} \boldsymbol{v}_{\tau}$, and $\sigma(\boldsymbol{u}) \boldsymbol{n v}=[\sigma(\boldsymbol{u}) \boldsymbol{n}]_{\boldsymbol{n}} \boldsymbol{v}_{\boldsymbol{n}}+[\sigma(\boldsymbol{u}) \boldsymbol{n}]_{\tau} \boldsymbol{v}_{\tau}$. Hence (4) becomes

$$
\int_{\Omega} 2 \nu \mathbf{D}(\boldsymbol{u}): \mathbf{D}(\boldsymbol{v})-\int_{\Omega} p \nabla \cdot \boldsymbol{v}=\int_{\partial \Omega}[\sigma(\boldsymbol{u}) \boldsymbol{n}]_{\boldsymbol{n}} \boldsymbol{v}_{\boldsymbol{n}}+\int_{\partial \Omega}[\sigma(\boldsymbol{u}) \boldsymbol{n}]_{\tau} \boldsymbol{v}_{\tau} .
$$

The Sobolev space that contains the velocity field which fulfill the penetration condition as an essential part of the boundary conditions of (2) is denoted by

$H_{\text {ess }}^{1}(\Omega):=\left\{\boldsymbol{u} \in\left(H^{1}(\Omega)\right)^{d}\right.$, s. t. $\left.\boldsymbol{u}\right|_{\Gamma_{s}}=0$ and $\left.\boldsymbol{u} \cdot \boldsymbol{n}\right|_{\Gamma_{c}}=0$ in the sense of traces $\}$.

The mixed weak formulation of problem (2) considered in [22, 25] seeks $(\boldsymbol{u}, p) \in H_{\text {ess }}^{1}(\Omega) \times$ $L_{0}^{2}(\Omega)$ such that $\boldsymbol{u}=\varphi$ on $\Gamma_{i}$ and

$$
\begin{aligned}
\int_{\Omega} 2 \nu \mathbf{D}(\boldsymbol{u}): \mathbf{D}(\boldsymbol{v})-\int_{\Omega} p \nabla \cdot \boldsymbol{v} & =\int_{\Gamma_{c}} T \boldsymbol{v}_{\tau}, \quad \forall \boldsymbol{v} \in H_{\text {ess }}^{1}(\Omega), \\
\int_{\Omega} \nabla \cdot \boldsymbol{u} q & =0, \quad \forall q \in L_{0}^{2}(\Omega),
\end{aligned}
$$

and its well-posedness is given by the following theorem [22].

Theorem 3 Suppose that the following assumptions hold: $\overline{\Gamma_{i}} \cap \overline{\Gamma_{c}} \cap \overline{\Gamma_{s}}=\emptyset$, measure $\left(\Gamma_{i}\right)>$ 0 , and $T \in V^{-1}\left(\Gamma_{c}\right)$. Then there exists a unique solution $(\boldsymbol{u}, p) \in H_{\text {ess }}^{1}(\Omega) \times L_{0}^{2}(\Omega)$ of (8)-(9). Moreover, the regularity estimate

$$
\|\boldsymbol{u}\|_{H^{1}(\Omega)}+\|p\|_{L^{2}(\Omega)} \leq C\left\{\|T\|_{V^{-1}\left(\Gamma_{c}\right)}+\|\varphi\|_{V^{1}\left(\Gamma_{i}\right)}\right\}
$$

holds for some constant $C>0$.

Note that in case of $\left|\Gamma_{c}\right|=0$ (that is $\Gamma_{c}$ is of measure zero), this problem is not uniquely solvable and the solution $(\boldsymbol{u}, p)$ is only unique up to a rigid body motion and belongs to $H_{\text {ess }}^{1}(\Omega) \cap \Re$ where

$$
\Re=\left\{\mathbf{A} x+b \mid \mathbf{A} \in \mathbb{R}^{d \times d} \text { skew symmetric }, b \in \mathbb{R}^{d}\right\} .
$$




\section{Energy-like minimization method}

The approach followed here extends the one given in [7] to the partially overdetermined Cauchy system. More precisely, we focus on a method based on the minimization of an energy-like functional. This approach in which two distinct fields are introduced, each of them representing only one item of the given data has to be adapted to our formulation. In our case, in lack of complete Neumann boundary condition it is really important to introduce another boundary conditions on $\Gamma_{c}$ in order to obtain two different well-posed problems. More precisely, the Dirichlet boundary data can be separated into two parts :

$$
\left\{\begin{array}{cl}
\boldsymbol{u} \cdot \boldsymbol{n}=\Phi \cdot \boldsymbol{n}, & \text { on } \Gamma_{c} \\
\boldsymbol{u} \cdot \tau=\Phi \cdot \tau, & \text { on } \Gamma_{c}
\end{array}\right.
$$

and the normal component part $\boldsymbol{u} \cdot \boldsymbol{n}$ will be posed as an essential boundary condition. For what follows we consider $\Phi \cdot \boldsymbol{n}=0$ and we introduce two distinct fields $\left(\boldsymbol{u}_{1}, p_{1}\right)$ and $\left(\boldsymbol{u}_{2}, p_{2}\right)$ solutions of problems which differ by their boundary conditions and meeting some of the existing boundary data: We attribute to the first problem a Dirichlet boundary data on $\Gamma_{c}$ and one unknown on $\Gamma_{i}$, while we attribute to the second one Navier boundary condition given shear stress and penetration conditions on $\Gamma_{c}$ and one unknown on $\Gamma_{i}$. Hence, given pair $(g, \eta) \in V^{1}\left(\Gamma_{c}\right)^{2} \times V^{-1}\left(\Gamma_{c}\right)^{2}$, we obtain the following mixed boundary value problems

$$
\left(P_{D}\right)\left\{\begin{array} { l l } 
{ - \nu \Delta \boldsymbol { u } _ { 1 } ^ { \eta } + \nabla p _ { 1 } ^ { \eta } = 0 } & { \text { in } \Omega } \\
{ \nabla \cdot \boldsymbol { u } _ { 1 } ^ { \eta } = 0 } & { \text { in } \Omega } \\
{ \boldsymbol { u } _ { 1 } ^ { \eta } = 0 } & { \text { on } \Gamma _ { s } ( P _ { N } ) } \\
{ \boldsymbol { u } _ { 1 } ^ { \eta } = \Phi } & { \text { on } \Gamma _ { c } } \\
{ \boldsymbol { \sigma } ( \boldsymbol { u } _ { 1 } ^ { \eta } ) \boldsymbol { n } + \alpha \boldsymbol { u } _ { 1 } ^ { \eta } = \eta + \alpha g } & { \text { on } \Gamma _ { i } }
\end{array} \left\{\begin{array}{ll}
-\nu \Delta \boldsymbol{u}_{2}^{g}+\nabla p_{2}^{g}=0 & \text { in } \Omega \\
\nabla \cdot \boldsymbol{u}_{2}^{g}=0 & \text { in } \Omega \\
\boldsymbol{u}_{2}^{g}=0 & \text { on } \Gamma_{s} \\
{\left[\boldsymbol{\sigma}\left(\boldsymbol{u}_{2}^{g}\right) \boldsymbol{n}\right]_{\tau}=T} & \text { on } \Gamma_{c} \\
\boldsymbol{u}_{2}^{g} \cdot \boldsymbol{n}=0 & \text { on } \Gamma_{c} \\
\boldsymbol{\sigma}\left(\boldsymbol{u}_{2}^{g}\right) \boldsymbol{n}+\beta \boldsymbol{u}_{2}^{g}=\eta+\beta g & \text { on } \Gamma_{i}
\end{array}\right.\right.
$$

where we denote by $\alpha$ and $\beta$ two non-negative real coefficients that permit to define various approaches that differ by the number of unknown fields on $\Gamma_{c}$. Note that the existence and uniqueness of the solution of the two problems is guaranteed by Theorem 3 for $\left(P_{N}\right)$ and by [4] for $\left(P_{D}\right)$.

In what follows, we will show how it is possible to prescribe the shear stress and the normal velocity forming Navier boundary condition for recovering the boundary data on $\Gamma_{i}$ [5]. We consider now the following energy-like functional in order to compare the two fields $\left(\boldsymbol{u}_{1}^{\eta}, p_{1}^{\eta}\right)$ and $\left(\boldsymbol{u}_{2}^{g}, p_{2}^{g}\right)$

$$
E_{\alpha \beta}(g, \eta)=\frac{1}{2} \int_{\Omega} \boldsymbol{\sigma}\left(\boldsymbol{u}_{1}^{\eta}-\boldsymbol{u}_{2}^{g}\right): \nabla\left(\boldsymbol{u}_{1}^{\eta}-\boldsymbol{u}_{2}^{g}\right)
$$


where they are equal only when the pair $(g, \eta)$ meets the real data $(\varphi, t)$ on the boundary $\Gamma_{i}$. Hence, the desired data $(\varphi, t)$ can be characterized as the solution of the following minimization problem :

$$
(\varphi, t)=\underset{(g, \eta)}{\arg \min } E_{\alpha \beta}(g, \eta),
$$

with $g \in V^{1}\left(\Gamma_{i}\right)^{2}$ and $\eta \in V^{-1}\left(\Gamma_{i}\right)^{2}$. Throughout the paper we will treat the minimization problem using Neumann-Dirichlet approach, $(\alpha=0, \beta=\infty)$ denoted by ND, which corresponds to consider $\left(P_{D}\right)$ with Neumann boundary condition on $\Gamma_{i}$, and $\left(P_{N}\right)$ with Dirichlet boundary condition on $\Gamma_{i}$. The Dirichlet-Dirichlet approach $(\alpha=\beta=\infty)$ which will be denoted by DD, corresponds to consider $\left(P_{D}\right)$ and $\left(P_{N}\right)$ with the same unknown Dirichlet boundary condition on $\Gamma_{i}$. The third approach is Neumann-Neumann approach denoted by NN $(\alpha=\beta=0)$ and where we consider $\left(P_{D}\right)$ and $\left(P_{N}\right)$ with the same unknown Neumann boundary condition on $\Gamma_{i}$.

\subsection{Neumann-Dirichlet case :}

We consider two mixed well-posed problems : the first one is a classical Dirichlet problem $\left(P_{D}\right)$ (with Dirichlet condition on $\Gamma_{c}$ ), and the second one is a Stokes problem with Navier boundary condition on $\Gamma_{c}$. We attribute to each of them one unknown on $\Gamma_{i}$ i.e. (13) with unknown Neumann boundary condition on $\Gamma_{i}$, and (14) with unknown Dirichlet boundary condition on $\Gamma_{i}$ :

$$
\left\{\begin{array} { l l } 
{ - \nu \Delta \boldsymbol { u } _ { 1 } ^ { \eta } + \nabla p _ { 1 } ^ { \eta } = 0 } & { \text { in } \Omega } \\
{ \nabla \cdot \boldsymbol { u } _ { 1 } ^ { \eta } = 0 } & { \text { in } \Omega } \\
{ \boldsymbol { u } _ { 1 } ^ { \eta } = 0 } & { \text { on } \Gamma _ { s } \quad ( 1 3 ) } \\
{ \boldsymbol { u } _ { 1 } ^ { \eta } = \Phi } & { \text { on } \Gamma _ { c } } \\
{ \boldsymbol { \sigma } ( \boldsymbol { u } _ { 1 } ^ { \eta } ) \boldsymbol { n } = \eta } & { \text { on } \Gamma _ { i } }
\end{array} \quad \left\{\begin{array}{ll}
-\nu \Delta \boldsymbol{u}_{2}^{g}+\nabla p_{2}^{g}=0 & \text { in } \Omega \\
\nabla \cdot \boldsymbol{u}_{2}^{g}=0 & \text { in } \Omega \\
\boldsymbol{u}_{2}^{g}=0 & \text { on } \Gamma_{s} \\
{\left[\boldsymbol{\sigma}\left(\boldsymbol{u}_{2}^{g}\right) \boldsymbol{n}\right]_{\tau}=T} & \text { on } \Gamma_{c} \\
\boldsymbol{u}_{2}^{g} \cdot \boldsymbol{n}=0 & \text { on } \Gamma_{c} \\
\boldsymbol{u}_{2}^{g}=g & \text { on } \Gamma_{i}
\end{array}\right.\right.
$$

In this case, the functional (11) depends on the pair $(g, \eta)$

$$
E_{N D}(g, \eta)=\frac{1}{2} \int_{\Omega} \boldsymbol{\sigma}\left(\boldsymbol{u}_{1}^{\eta}-\boldsymbol{u}_{2}^{g}\right): \nabla\left(\boldsymbol{u}_{1}^{\eta}-\boldsymbol{u}_{2}^{g}\right)
$$

Then, the gradient of $E_{N D}$ can be obtained from its partial derivatives with respect to $g$ and $\eta$, that is

Proposition 4 For a pair $(g, \eta) \in V^{1}\left(\Gamma_{i}\right)^{2} \times V^{-1}\left(\Gamma_{i}\right)^{2}$

$$
\begin{aligned}
\frac{\partial E_{N D}(g, \eta)}{\partial \eta} \cdot h & =2 \nu \int_{\Omega} \mathbf{D}\left(\boldsymbol{u}_{1}^{\eta}-\boldsymbol{u}_{2}^{g}\right): \nabla \boldsymbol{r}_{1}^{h} \\
& =\int_{\Gamma_{i}} \boldsymbol{\sigma}\left(\boldsymbol{u}_{1}^{\eta}-\boldsymbol{u}_{2}^{g}\right) \boldsymbol{n} \boldsymbol{r}_{1}^{h}, \quad \forall h \in V^{-1}\left(\Gamma_{i}\right)^{2}, \\
\frac{\partial E_{N D}(g, \eta)}{\partial g} \cdot w & =-2 \nu \int_{\Omega} \mathbf{D}\left(\boldsymbol{r}_{2}^{w}\right): \nabla\left(\boldsymbol{u}_{1}^{\eta}-\boldsymbol{u}_{2}^{g}\right) \\
& =-\int_{\Gamma_{i}} \boldsymbol{\sigma}\left(\boldsymbol{r}_{2}^{w}\right) \boldsymbol{n}\left(\boldsymbol{u}_{1}^{\eta}-\boldsymbol{u}_{2}^{g}\right), \quad \forall w \in V^{1}\left(\Gamma_{i}\right)^{2},
\end{aligned}
$$


where $\left(\boldsymbol{r}_{1}^{h}, s_{1}^{h}\right)$ and $\left(\boldsymbol{r}_{2}^{w}, s_{2}^{w}\right)$ are the solution of

$$
\left\{\begin{array} { l l } 
{ - \nu \Delta \boldsymbol { r } _ { 1 } ^ { h } + \nabla s _ { 1 } ^ { h } = 0 } & { \text { in } \Omega } \\
{ \nabla \cdot \boldsymbol { r } _ { 1 } ^ { h } = 0 } & { \text { in } \Omega } \\
{ \boldsymbol { r } _ { 1 } ^ { h } = 0 } & { \text { on } \Gamma _ { s } } \\
{ \boldsymbol { r } _ { 1 } ^ { h } = 0 } & { \text { on } \Gamma _ { c } } \\
{ \boldsymbol { \sigma } ( \boldsymbol { r } _ { 1 } ^ { h } ) \boldsymbol { n } = h } & { \text { on } \Gamma _ { i } }
\end{array} \quad \left\{\begin{array}{ll}
-\nu \Delta \boldsymbol{r}_{2}^{w}+\nabla s_{2}^{w}=0 & \text { in } \Omega \\
\nabla \cdot \boldsymbol{r}_{2}^{w}=0 & \text { in } \Omega \\
\boldsymbol{r}_{2}^{w}=0 & \text { on } \Gamma_{s} \\
{\left[\boldsymbol{\sigma}\left(\boldsymbol{r}_{2}^{w}\right) \boldsymbol{n}\right]_{\tau}=0} & \text { on } \Gamma_{c} \\
\boldsymbol{r}_{2}^{w} \cdot \boldsymbol{n}=0 & \text { on } \Gamma_{c} \\
\boldsymbol{r}_{2}^{w}=w & \text { on } \Gamma_{i}
\end{array}\right.\right.
$$

Proof: We easily derive the partial derivative of $E_{N D}$ with respect to $\eta$

$\frac{\partial E_{N D}(g, \eta)}{\partial \eta} \cdot h=2 \nu \int_{\Omega} \mathbf{D}\left(\boldsymbol{u}_{1}^{\eta}-\boldsymbol{u}_{2}^{g}\right): \nabla \boldsymbol{r}_{1}^{h}=\int_{\partial \Omega} \boldsymbol{\sigma}\left(\boldsymbol{u}_{1}^{\eta}-\boldsymbol{u}_{2}^{g}\right) \boldsymbol{n} \boldsymbol{r}_{1}^{h}, \quad \forall h \in V^{-1}\left(\Gamma_{i}\right)^{2}$,

using that $\boldsymbol{r}_{1}^{h}=0$ on $\Gamma_{c} \cup \Gamma_{s}$, then the first derivative holds.

Now, using the Green formula we obtain, $\forall w \in V^{1}\left(\Gamma_{i}\right)^{2}$ :

$$
\frac{\partial E_{N D}(g, \eta)}{\partial g} \cdot w=-2 \nu \int_{\Omega} \mathbf{D}\left(\boldsymbol{r}_{2}^{w}\right): \nabla\left(\boldsymbol{u}_{1}^{\eta}-\boldsymbol{u}_{2}^{g}\right)=-\int_{\partial \Omega} \boldsymbol{\sigma}\left(\boldsymbol{r}_{2}^{w}\right) \boldsymbol{n}\left(\boldsymbol{u}_{1}^{\eta}-\boldsymbol{u}_{2}^{g}\right),
$$

thanks to (5), we obtain

$$
\begin{gathered}
\frac{\partial E_{N D}(g, \eta)}{\partial g} \cdot w=-\int_{\Gamma_{i}} \boldsymbol{\sigma}\left(\boldsymbol{r}_{2}^{w}\right) \boldsymbol{n}\left(\boldsymbol{u}_{1}^{\eta}-\boldsymbol{u}_{2}^{g}\right)-\int_{\Gamma_{c}}\left[\boldsymbol{\sigma}\left(\boldsymbol{r}_{2}^{w}\right) \boldsymbol{n}\right]_{\boldsymbol{n}}\left(\boldsymbol{u}_{1}^{\eta}-\boldsymbol{u}_{2}^{g}\right)_{\boldsymbol{n}} \\
-\int_{\Gamma_{c}}\left[\boldsymbol{\sigma}\left(\boldsymbol{r}_{2}^{w}\right) \boldsymbol{n}\right]_{\tau}\left(\boldsymbol{u}_{1}^{\eta}-\boldsymbol{u}_{2}^{g}\right)_{\tau},
\end{gathered}
$$

then, since $\left(\boldsymbol{u}_{1}^{\eta}-\boldsymbol{u}_{2}^{g}\right)_{n}=0$ and $\left[\boldsymbol{\sigma}\left(\boldsymbol{r}_{2}^{w}\right) \boldsymbol{n}\right]_{\tau}=0$ on $\Gamma_{c}$, the second derivative in (16) follows.

\subsection{Dirichlet-Dirichlet case :}

In this case, we consider two well-posed problems with the same unknown Dirichlet condition on $\Gamma_{i}$.

$$
\left\{\begin{array} { l l } 
{ - \nu \Delta \boldsymbol { u } _ { 1 } ^ { g } + \nabla p _ { 1 } ^ { g } = 0 } & { \text { in } \Omega } \\
{ \nabla \cdot \boldsymbol { u } _ { 1 } ^ { g } = 0 } & { \text { in } \Omega } \\
{ \boldsymbol { u } _ { 1 } ^ { g } = 0 } & { \text { on } \Gamma _ { s } } \\
{ \boldsymbol { u } _ { 1 } ^ { g } = \Phi } & { \text { on } \Gamma _ { c } } \\
{ \boldsymbol { u } _ { 1 } ^ { g } = g } & { \text { on } \Gamma _ { i } }
\end{array} \quad \left\{\begin{array}{ll}
-\nu \Delta \boldsymbol{u}_{2}^{g}+\nabla p_{2}^{g}=0 & \text { in } \Omega \\
\nabla \cdot \boldsymbol{u}_{2}^{g}=0 & \text { in } \Omega \\
\boldsymbol{u}_{2}^{g}=0 & \text { on } \Gamma_{s} \\
{\left[\boldsymbol{\sigma}\left(\boldsymbol{u}_{2}^{g}\right) \boldsymbol{n}\right]_{\tau}=T} & \text { on } \Gamma_{c} \\
\boldsymbol{u}_{2}^{g} \cdot \boldsymbol{n}=0 & \text { on } \Gamma_{c} \\
\boldsymbol{u}_{2}^{g}=g & \text { on } \Gamma_{i}
\end{array}\right.\right.
$$

In this case, the functional (11) depends only on the unknown trace $g$ :

$$
E_{D D}(g)=\frac{1}{2} \int_{\Omega} \boldsymbol{\sigma}\left(\boldsymbol{u}_{1}^{g}-\boldsymbol{u}_{2}^{g}\right): \nabla\left(\boldsymbol{u}_{1}^{g}-\boldsymbol{u}_{2}^{g}\right)
$$

Analogously, we derive the partial derivative of $E_{D D}$ with respect to $g$ 
Proposition 5 For $g \in V^{1}\left(\Gamma_{c}\right)^{2}$

$$
\frac{\partial E_{D D}(g)}{\partial g} \cdot h=\frac{1}{2} \int_{\Gamma_{i}} \boldsymbol{\sigma}\left(\boldsymbol{u}_{1}^{g}-\boldsymbol{u}_{2}^{g}\right) \boldsymbol{n} h \quad \forall h \in V^{1}\left(\Gamma_{i}\right)^{2},
$$

where $\left(\boldsymbol{r}_{1}^{h}, s_{1}^{h}\right)$ and $\left(\boldsymbol{r}_{2}^{h}, s_{2}^{h}\right)$ are the respective solutions of

$$
\left\{\begin{array} { l l } 
{ - \nu \Delta \boldsymbol { r } _ { 1 } ^ { h } + \nabla s _ { 1 } ^ { h } = 0 } & { \text { in } \Omega } \\
{ \nabla \cdot \boldsymbol { r } _ { 1 } ^ { h } = 0 } & { \text { in } \Omega } \\
{ \boldsymbol { r } _ { 1 } ^ { h } = 0 } & { \text { on } \Gamma _ { s } } \\
{ \boldsymbol { r } _ { 1 } ^ { h } = 0 } & { \text { on } \Gamma _ { c } } \\
{ \boldsymbol { r } _ { 1 } ^ { h } = h } & { \text { on } \Gamma _ { i } }
\end{array} \quad \left\{\begin{array}{ll}
-\nu \Delta \boldsymbol{r}_{2}^{h}+\nabla s_{2}^{h}=0 & \text { in } \Omega \\
\nabla \cdot \boldsymbol{r}_{2}^{h}=0 & \text { in } \Omega \\
\boldsymbol{r}_{2}^{h}=0 & \text { on } \Gamma_{s} \\
{\left[\boldsymbol{\sigma}\left(\boldsymbol{r}_{2}^{h}\right) \boldsymbol{n}\right]_{\tau}=0} & \text { on } \Gamma_{c} \\
\boldsymbol{r}_{2}^{h} \cdot \boldsymbol{n}=0 & \text { on } \Gamma_{c} \\
\boldsymbol{r}_{2}^{h}=h & \text { on } \Gamma_{i}
\end{array}\right.\right.
$$

Proof: The weak formulation of the adjoint problems leads to

$\frac{\partial E_{D D}(g)}{\partial g} \cdot h=\frac{1}{2} \int_{\partial \Omega} \boldsymbol{\sigma}\left(\boldsymbol{u}_{1}^{g}-\boldsymbol{u}_{2}^{g}\right) \boldsymbol{n} \boldsymbol{r}_{1}^{h}-\frac{1}{2} \int_{\partial \Omega}\left(\boldsymbol{u}_{1}^{g}-\boldsymbol{u}_{2}^{g}\right) \boldsymbol{\sigma}\left(\boldsymbol{r}_{2}^{h}\right) \boldsymbol{n}, \quad \forall h \in V^{1}\left(\Gamma_{i}\right)^{2}$,

using that $\boldsymbol{r}_{1}^{h}=0$ on $\Gamma_{c} \cup \Gamma_{s}$, and $\left(\boldsymbol{u}_{1}^{g}-\boldsymbol{u}_{2}^{g}\right) \cdot \boldsymbol{n}=0$ on $\Gamma_{c}$ then we obtain

$\frac{\partial E_{D D}(g)}{\partial g} \cdot h=\frac{1}{2} \int_{\Gamma_{i}} \boldsymbol{\sigma}\left(\boldsymbol{u}_{1}^{g}-\boldsymbol{u}_{2}^{g}\right) \boldsymbol{n} \boldsymbol{r}_{1}^{h}-\frac{1}{2} \int_{\Gamma_{c}}\left(\boldsymbol{u}_{1}^{g}-\boldsymbol{u}_{2}^{g}\right) \cdot \tau\left[\boldsymbol{\sigma}\left(\boldsymbol{r}_{2}^{h}\right) \boldsymbol{n}\right]_{\tau}, \quad \forall h \in V^{1}\left(\Gamma_{i}\right)^{2}$,

then using $\left[\boldsymbol{\sigma}\left(\boldsymbol{r}_{2}^{h}\right) \boldsymbol{n}\right]_{\tau}=0$, the derivative of $E_{D D}$ with respect to $g$ is

$$
\frac{\partial E_{D D}(g)}{\partial g} \cdot h=\frac{1}{2} \int_{\Gamma_{i}} \boldsymbol{\sigma}\left(\boldsymbol{u}_{1}^{g}-\boldsymbol{u}_{2}^{g}\right) \boldsymbol{n} h, \quad \forall h \in V^{1}\left(\Gamma_{i}\right)^{2} .
$$

\subsection{Neumann-Neumann case :}

In this case, we impose unknown Neumann condition on $\Gamma_{i}$. We consider the following mixed boundary value problems

$$
\left\{\begin{array} { l l } 
{ - \nu \Delta \boldsymbol { u } _ { 1 } ^ { \eta } + \nabla p _ { 1 } ^ { \eta } = 0 } & { \text { in } \Omega } \\
{ \nabla \cdot \boldsymbol { u } _ { 1 } ^ { \eta } = 0 } & { \text { in } \Omega } \\
{ \boldsymbol { u } _ { 1 } ^ { \eta } = 0 } & { \text { on } \Gamma _ { s } } \\
{ \boldsymbol { u } _ { 1 } ^ { \eta } = \Phi } & { \text { on } \Gamma _ { c } } \\
{ \boldsymbol { \sigma } ( \boldsymbol { u } _ { 1 } ^ { \eta } ) \boldsymbol { n } = \eta } & { \text { on } \Gamma _ { i } }
\end{array} \quad \left\{\begin{array}{ll}
-\nu \Delta \boldsymbol{u}_{2}^{\eta}+\nabla p_{2}^{\eta}=0 & \text { in } \Omega \\
\nabla \cdot \boldsymbol{u}_{2}^{\eta}=0 & \text { in } \Omega \\
\boldsymbol{u}_{2}^{\eta}=0 & \text { on } \Gamma_{s} \\
{\left[\boldsymbol{\sigma}\left(\boldsymbol{u}_{2}^{\eta}\right) \boldsymbol{n}\right]_{\tau}=T} & \text { on } \Gamma_{c} \\
\boldsymbol{u}_{2}^{\eta} \cdot \boldsymbol{n}=0 & \text { on } \Gamma_{c} \\
\boldsymbol{\sigma}\left(\boldsymbol{u}_{2}^{\eta}\right) \boldsymbol{n}=\eta & \text { on } \Gamma_{i}
\end{array}\right.\right.
$$

Let us recall that here the functional (11) depends only on the variable $\eta$ :

$$
E_{N N}(\eta)=\frac{1}{2} \int_{\Omega} \boldsymbol{\sigma}\left(\boldsymbol{u}_{1}^{\eta}-\boldsymbol{u}_{2}^{\eta}\right): \nabla\left(\boldsymbol{u}_{1}^{\eta}-\boldsymbol{u}_{2}^{\eta}\right)
$$

The gradient of $E_{N N}$ is then 
Proposition 6 For $\eta \in V^{-1}\left(\Gamma_{c}\right)^{2}$

$$
\frac{\partial E_{N N}(\eta)}{\partial \eta} \cdot \psi=-\frac{1}{2} \int_{\Gamma_{i}}\left(\boldsymbol{u}_{1}^{\eta}-\boldsymbol{u}_{2}^{\eta}\right) \psi, \quad \forall \psi \in V^{-1}\left(\Gamma_{i}\right)^{2},
$$

and where $\left(\boldsymbol{r}_{1}^{\psi}, s_{1}^{\psi}\right)$ and $\left(\boldsymbol{r}_{2}^{\psi}, s_{2}^{\psi}\right)$ solves

$$
\left\{\begin{array} { l l } 
{ - \nu \Delta \boldsymbol { r } _ { 1 } ^ { \psi } + \nabla s _ { 1 } ^ { \psi } = 0 } & { \text { in } \Omega } \\
{ \nabla \cdot \boldsymbol { r } _ { 1 } ^ { \psi } = 0 } & { \text { in } \Omega } \\
{ \boldsymbol { r } _ { 1 } ^ { \psi } = 0 } & { \text { on } \Gamma _ { s } } \\
{ \boldsymbol { r } _ { 1 } ^ { \psi } = 0 } & { \text { on } \Gamma _ { c } } \\
{ \boldsymbol { \sigma } ( \boldsymbol { r } _ { 1 } ^ { \psi } ) \boldsymbol { n } = \psi } & { \text { on } \Gamma _ { i } }
\end{array} \quad \left\{\begin{array}{ll}
-\nu \Delta \boldsymbol{r}_{2}^{\psi}+\nabla s_{2}^{\psi}=0 & \text { in } \Omega \\
\nabla \cdot \boldsymbol{r}_{2}^{\psi}=0 & \text { in } \Omega \\
\boldsymbol{r}_{2}^{\psi}=0 & \text { on } \Gamma_{s} \\
{\left[\boldsymbol{\sigma}\left(\boldsymbol{r}_{2}^{\psi}\right) \boldsymbol{n}\right]_{\tau}=0} & \text { on } \Gamma_{c} \\
\boldsymbol{r}_{2}^{\psi} \cdot \boldsymbol{n}=0 & \text { on } \Gamma_{c} \\
\boldsymbol{\sigma}\left(\boldsymbol{r}_{2}^{\psi}\right) \boldsymbol{n}=\psi & \text { on } \Gamma_{i}
\end{array}\right.\right.
$$

Proof: The weak formulation of the adjoint problems leads, $\forall \psi \in V^{-1}\left(\Gamma_{i}\right)^{2}$, to

$$
\frac{\partial E_{N N}(\eta)}{\partial \eta} \cdot \psi=\frac{1}{2} \int_{\partial \Omega} \boldsymbol{\sigma}\left(\boldsymbol{u}_{1}^{\eta}-\boldsymbol{u}_{2}^{\eta}\right) \boldsymbol{n} \boldsymbol{r}_{1}^{\psi}-\frac{1}{2} \int_{\partial \Omega}\left(\boldsymbol{u}_{1}^{\eta}-\boldsymbol{u}_{2}^{\eta}\right) \boldsymbol{\sigma}\left(\boldsymbol{r}_{2}^{\psi}\right) \boldsymbol{n},
$$

using that $\boldsymbol{r}_{1}^{\psi}=0$ on $\Gamma_{c} \cup \Gamma_{s},\left(\boldsymbol{u}_{1}^{\eta}-\boldsymbol{u}_{2}^{\eta}\right) \cdot \boldsymbol{n}=0$ on $\Gamma_{c}$ and $\boldsymbol{\sigma}\left(\boldsymbol{u}_{1}^{\eta}-\boldsymbol{u}_{2}^{\eta}\right) \boldsymbol{n}=0$ on $\Gamma_{i}$, we obtain

$$
\frac{\partial E_{N N}(\eta)}{\partial \eta} \cdot \psi=-\frac{1}{2} \int_{\Gamma_{i}}\left(\boldsymbol{u}_{1}^{\eta}-\boldsymbol{u}_{2}^{\eta}\right) \boldsymbol{\sigma}\left(\boldsymbol{r}_{2}^{\psi}\right) \boldsymbol{n}-\frac{1}{2} \int_{\Gamma_{c}}\left(\boldsymbol{u}_{1}^{\eta}-\boldsymbol{u}_{2}^{\eta}\right) \cdot \tau\left[\boldsymbol{\sigma}\left(\boldsymbol{r}_{2}^{\psi}\right) \boldsymbol{n}\right]_{\tau},
$$

finally using that $\left[\boldsymbol{\sigma}\left(\boldsymbol{r}_{2}^{\psi}\right) \boldsymbol{n}\right]_{\tau}=0$ on $\Gamma_{c}$, the derivative of $E_{N D}$ with respect to $\eta$ is

$$
\frac{\partial E_{N N}(\eta)}{\partial \eta} \cdot \psi=-\frac{1}{2} \int_{\Gamma_{i}}\left(\boldsymbol{u}_{1}^{\eta}-\boldsymbol{u}_{2}^{\eta}\right) \boldsymbol{\sigma}\left(\boldsymbol{r}_{2}^{\psi}\right) \boldsymbol{n}
$$

Remark 7 We can show that in case of lack of one component of the the normal stress on the accessible boundary $\Gamma_{c}$, the gradient of the functional in the different approaches is still expressed by an integral involving only the boundary $\Gamma_{i}$. The same results hold when available data refered to the Dirichlet data with the normal component of the normal stress :

$$
\boldsymbol{u}=\Phi \text { and }[\boldsymbol{\sigma}(\boldsymbol{u}) \boldsymbol{n}]_{\boldsymbol{n}}=T \quad \text { on } \Gamma_{c} .
$$

Similar changes need to be made when using these data. The Neumann problem $\left(P_{N}\right)$ should be modified consequently, and we impose the tangential component of velocity $\boldsymbol{u}_{\tau}=\Phi_{\tau}$ with $[\boldsymbol{\sigma}(\boldsymbol{u}) \boldsymbol{n}]_{\boldsymbol{n}}=T$ as boundary conditions.

Theorem 8 The functional $(g, \eta) \longmapsto E_{N D}(g, \eta)$ defined by (15) is a positive quadratic functional. It's strictly convex on $V^{1}\left(\Gamma_{i}\right)^{2} \times V^{-1}\left(\Gamma_{i}\right)^{2}$ and consequently has a unique minimum on $V^{1}\left(\Gamma_{i}\right)^{2} \times V^{-1}\left(\Gamma_{i}\right)^{2}$ for a compatible data $(\Phi, T)$. 
Proof : First, since $\nabla \cdot\left(\boldsymbol{u}_{1}^{\eta}-\boldsymbol{u}_{2}^{g}\right)=0$, we can write :

$$
E_{N D}(g, \eta)=\nu \int_{\Omega} \mathbf{D}\left(\boldsymbol{u}_{1}^{\eta}-\boldsymbol{u}_{2}^{g}\right): \mathbf{D}\left(\boldsymbol{u}_{1}^{\eta}-\boldsymbol{u}_{2}^{g}\right)
$$

which shows the positive quadratic aspect of $E_{N D}$.

Recall that the derivative of $E_{N D}$ with respect to $g$ is given by (16) :

$$
\frac{\partial E_{N D}(g, \eta)}{\partial g} \cdot w=-2 \nu \int_{\Omega} \mathbf{D}\left(\boldsymbol{r}_{2}^{w}\right): \nabla\left(\boldsymbol{u}_{1}^{\eta}-\boldsymbol{u}_{2}^{g}\right)
$$

where $\boldsymbol{r}_{2}^{w}$ is a solution of (18). The second derivative is then given by :

$$
\frac{\partial^{2} E_{N D}(g, \eta)}{\partial g^{2}}(w, w)=2 \nu \int_{\Omega} \mathbf{D}\left(\boldsymbol{r}_{2}^{w}\right): \mathbf{D}\left(\boldsymbol{r}_{2}^{w}\right)=2 \nu\left\|\mathbf{D}\left(\boldsymbol{r}_{2}^{w}\right)\right\|_{L^{2}(\Omega)}^{2}, \quad \forall w \in V^{1}\left(\Gamma_{i}\right)^{2},
$$

Using Korn inequality [18], we deduce that there exists a constant $C>0$ such that :

$$
\frac{\partial^{2} E_{N D}(g, \eta)}{\partial g^{2}}(w, w) \geq C\left\|\boldsymbol{r}_{2}^{w}\right\|_{H^{1}(\Omega)}^{2}
$$

and then

$$
\frac{\partial^{2} E_{N D}(g, \eta)}{\partial g^{2}}(w, w) \geq k\|w\|_{V^{1}\left(\Gamma_{i}\right)^{2}}^{2}
$$

for some constant $k>0$. This proves the strict convexity of $E_{N D}$ with respect to $g$. By the same way, using (16), we can write :

$$
\frac{\partial^{2} E_{N D}(g, \eta)}{\partial \eta^{2}}(h, h)=2 \nu\left\|\mathbf{D}\left(\boldsymbol{r}_{1}^{h}\right)\right\|_{L^{2}(\Omega)}^{2}, \quad \forall h \in V^{-1}\left(\Gamma_{i}\right)^{2}
$$

where $r_{1}^{h}$ is a solution of (17). This shows the convexity of $E_{N D}$ with respect to $\eta$. To prove the strict convexity, we use the boundary expression of $\frac{\partial E_{N D}(g, \eta)}{\partial \eta}$, we obtain :

$$
\frac{\partial^{2} E_{N D}(g, \eta)}{\partial \eta^{2}}(h, h)=\int_{\Gamma_{i}} \boldsymbol{\sigma}\left(\boldsymbol{r}_{1}^{h}\right) \boldsymbol{n} \boldsymbol{r}_{1}^{h}=\int_{\Gamma_{i}} h \boldsymbol{r}_{1}^{h}, \quad \forall h \in V^{-1}\left(\Gamma_{i}\right)^{2},
$$

consequently, if $\frac{\partial^{2} E_{N D}(g, \eta)}{\partial \eta^{2}}(h, h)=0, \forall h \in V^{-1}\left(\Gamma_{i}\right)^{2}$, we deduce that $\boldsymbol{r}_{1}^{h}=0$ on $\Gamma_{i}$. Since $\boldsymbol{r}_{1}^{h}$ is zero on $\Gamma_{s}$ and $\Gamma_{c}$ we deduce that it's a trivial solution of (17) in $\Omega$ and therefore $h=0$ in $V^{-1}\left(\Gamma_{i}\right)^{2}$. 
Remark 9 We prove in the same way the convexity of $E_{D D}$ and $E_{N N}$. The strict convexity in these two cases is not obvious due to lack of unique continuation result. Nevertheless, we have used the Dirichlet-Dirichlet and the Neumann-Neumann approach in the sequel and for the numerical procedure in order to have a full overview of the subject.

\subsection{The first order optimality condition}

We derive the first optimality conditions and we have the following result :

Theorem 10 With partially overspecified Cauchy data $(\Phi, T)$ but compatible, lets note the pair $(\varphi, t)$ solution of

$$
\begin{aligned}
& (\varphi, t)=\underset{(g, \eta)}{\arg \min } E_{N D}(g, \eta), \quad \text { and } \quad E_{N D}(\varphi, t)=0, \\
& \varphi=\arg \min E_{D D}(g), \quad \text { and } E_{D D}(\varphi)=0 \text {, } \\
& g \in V^{1}\left(\Gamma_{i}\right)^{2} \\
& t=\arg \min E_{N N}(\eta), \quad \text { and } \quad E_{N N}(t)=0 .
\end{aligned}
$$

When the functional $E_{\alpha \beta}$ reach its minimum, the solutions $\left(\boldsymbol{u}_{1}^{t}, p_{1}^{t}\right)$ and $\left(\boldsymbol{u}_{2}^{\varphi}, p_{2}^{\varphi}\right)$ verify :

$$
\left\{\begin{array}{l}
\boldsymbol{u}_{1}^{t}=\boldsymbol{u}_{2}^{\varphi}+K, \text { on } \quad \Gamma_{i} \\
\boldsymbol{\sigma}\left(\boldsymbol{u}_{1}^{t}\right) \boldsymbol{n}=\boldsymbol{\sigma}\left(\boldsymbol{u}_{2}^{\varphi}\right) \boldsymbol{n}, \text { on } \Gamma_{i},
\end{array}\right.
$$

where $K$ is a constant.

Proof: Neumann-Dirichlet case : We consider the Steklov-poincaré operator :

$$
\begin{aligned}
S_{1}: \quad V^{1}\left(\Gamma_{i}\right)^{2} / \mathbb{R} & \longrightarrow V^{-1}\left(\Gamma_{i}\right)^{2} \\
w & \longmapsto \boldsymbol{\sigma}\left(\boldsymbol{r}_{2}^{w}\right) \boldsymbol{n}
\end{aligned}
$$

where $\boldsymbol{r}_{2}^{w}$ is the solution of (18). It is straightforward to check that $S_{1}$ is an isomorphism. Using this argument, first condition follows from the equation (16). In a similar way, using the inverse of the Steklov-poincaré operator :

$$
\begin{aligned}
S_{2}^{-1}: V^{-1}\left(\Gamma_{i}\right)^{2} & \longrightarrow V^{1}\left(\Gamma_{i}\right)^{2} \\
h & \longmapsto r_{1}^{h}
\end{aligned}
$$

where $\boldsymbol{r}_{1}^{h}$ is the solution of (17), one gets from (16) that $\boldsymbol{\sigma}\left(\boldsymbol{u}_{1}^{\eta}-\boldsymbol{u}_{2}^{g}\right) \boldsymbol{n}=0$ on $\Gamma_{i}$. Dirichlet-Dirichlet case : In this case first condition follows from the assumption of the same unknown Dirichlet condition on $\Gamma_{i}$. Second condition is a simple result of condition (22).

Neumann-Neumann case : Equivalently, first condition follows from the assumption of the same unknown Neumann condition on $\Gamma_{i}$. Second condition is a simple result of condition (29). 


\section{The numerical procedure}

We propose a numerical procedure based on the preconditioned gradient algorithm for the reconstruction of the data on the inaccessible boundary $\Gamma_{i}$. The following iterative algorithm for the Neumann-Dirichlet case is in the same spirit of the algorithm given in [7] and detailed in [9] for the Dirichlet-Dirichlet or Neumann-Neumann cases.

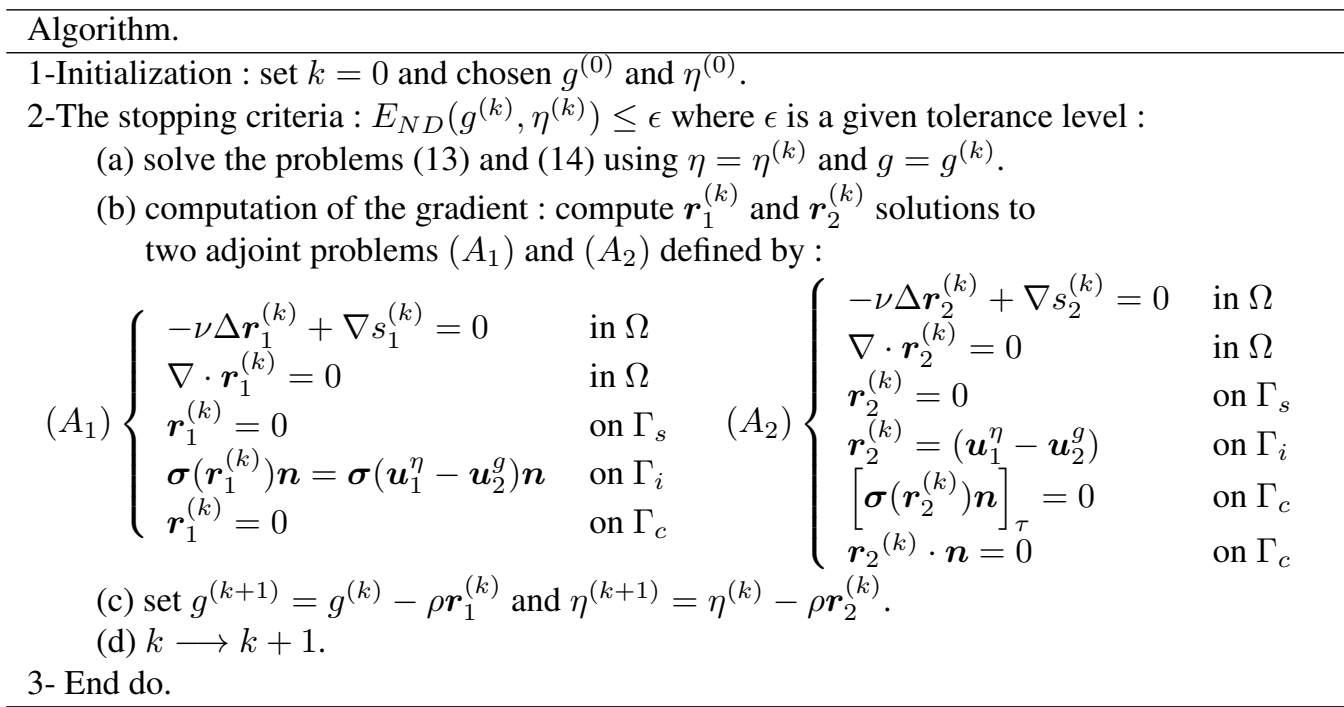

\section{Numerical results}

In this section, we will consider two test functions : a polynomial and a singular one. All the calculations are run under Freefem Software environement [23]. We consider $\Omega$ as a two-dimensional annular domain with radii $R_{1}=2$ and $R_{2}=1$. The outer boundary is chosen to be $\Gamma_{c}$ while the inner boundary is considered as $\Gamma_{i}$, as depicted in Figure 6. To explore the efficiency of the proposed approach procedure, we consider the reconstruction of the velocity field and the stress force on the inner circle form partially overdetermined data on the outer circle. 


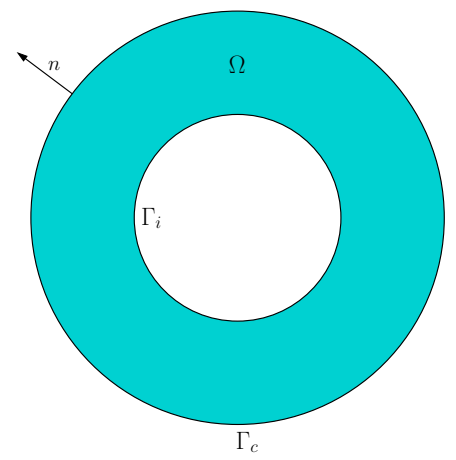

Figure 1. Computational domain

The minimization of the functional error is achieved by ensuring the optimality condition of the first order. To recover the velocity and the stress tensor with accuracy, a mesh with 50 nodes on $\Gamma_{i}$ is used.

\subsection{A Stokes flow in a ring with smooth data :}

In this test case we take a polynomial example, given by the following analytical function

$$
u(x, y):=\left(4 y^{3}-x^{2}, 4 x^{3}+2 x y-1\right), \quad p(x, y):=24 x y-2 x .
$$

Note that in this case $\boldsymbol{u} \cdot \boldsymbol{n} \neq 0$. Figures 2, 3 and 4 show the reconstructed Dirichlet and Neumann data on $\Gamma_{i}$ as well as the reconstructed normal component of the normal stress on $\Gamma_{c}$. They are compared with the exact data. Note that for the Neumann-Dirichlet and Neumann-Neumann, the reconstructed fields are in close agreement with the exact ones, while the Dirichlet-Dirichlet method gives worst result. 

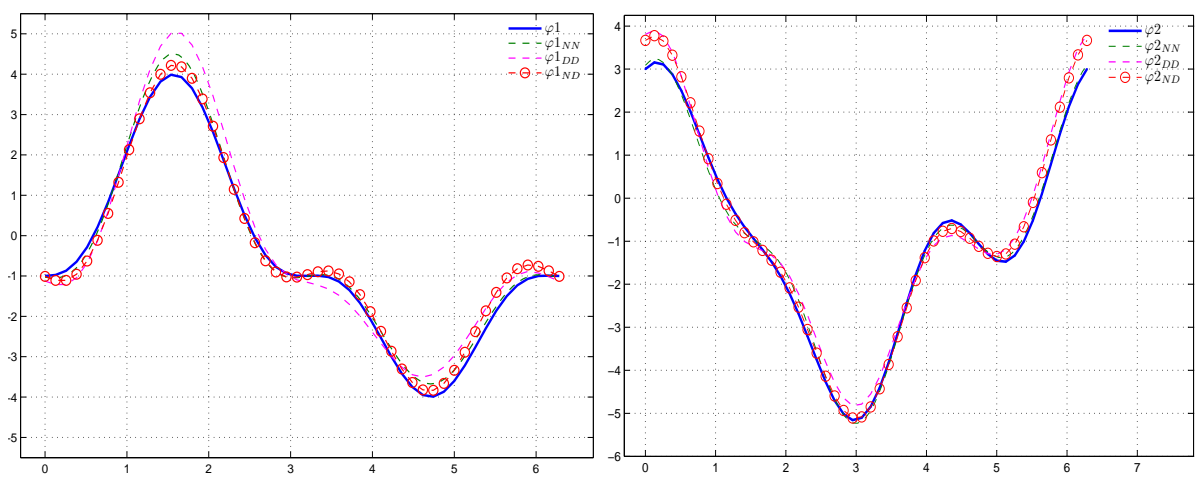

Figure 2. First test: The reconstructed velocity on $\Gamma_{i}$
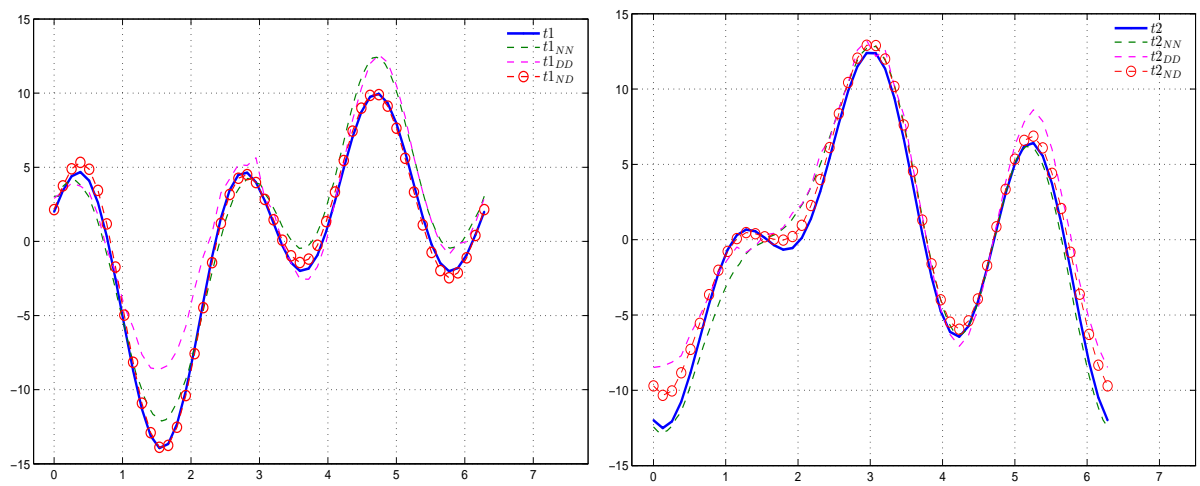

Figure 3. First test : The reconstructed stress tensor on $\Gamma_{i}$

\subsection{A Stokes flow in a ring with Singular data}

This example has already been addressed in [7] and it involves a singularity in the vicinity of the inner boundary.

$$
\begin{aligned}
& u(x, y)=\frac{1}{4 \pi}\left(\log \frac{1}{\sqrt{(x-a)^{2}+y^{2}}}+\frac{(x-a)^{2}}{(x-a)^{2}+y^{2}}, \frac{(x-a) y}{(x-a)^{2}+y^{2}}\right), \\
& p(x, y)=\frac{1}{2 \pi} \frac{(x-a)}{(x-a)^{2}+y^{2}} .
\end{aligned}
$$




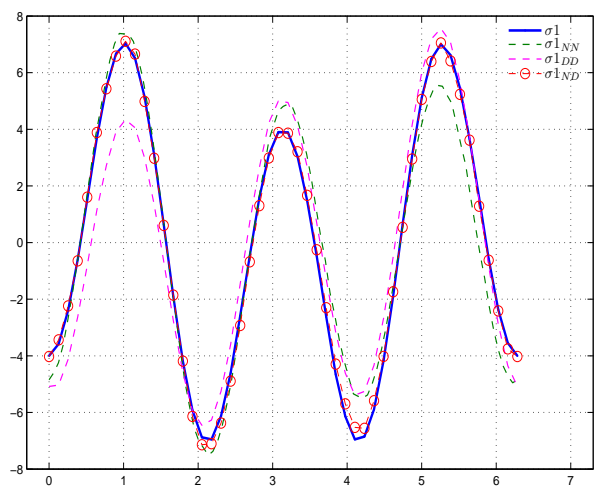

Figure 4. First test : The reconstructed stress on $\Gamma_{c}$

We have reconstructed the unknown data on $\Gamma_{i}$ using the Neumann-Dirichlet and Neumann-Neumann approaches. Figs 5 shows the obtained results. Note that the results are weaker than those obtained for complete Cauchy data, but the algorithm is still efficient in detecting the singularities.
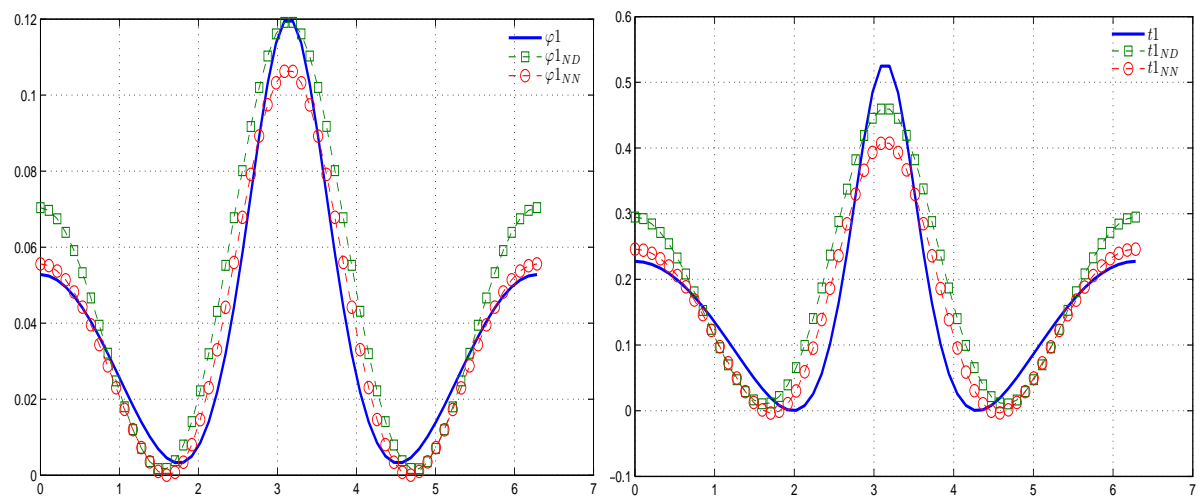

Figure 5. Singular data test : reconstructed velocity and the stress tensor on $\Gamma_{i}$ 
In order to test the robustness of the used method, we introduce a white noise perturbation to the data with an amplitude ranging from 1 to $15 \%$. We reconstruct the velocity and the stress tensor on $\Gamma_{i}$ from these noisy data. We observe in Figure6 that the method used is more robust with smooth data (left) than with singular one (right).
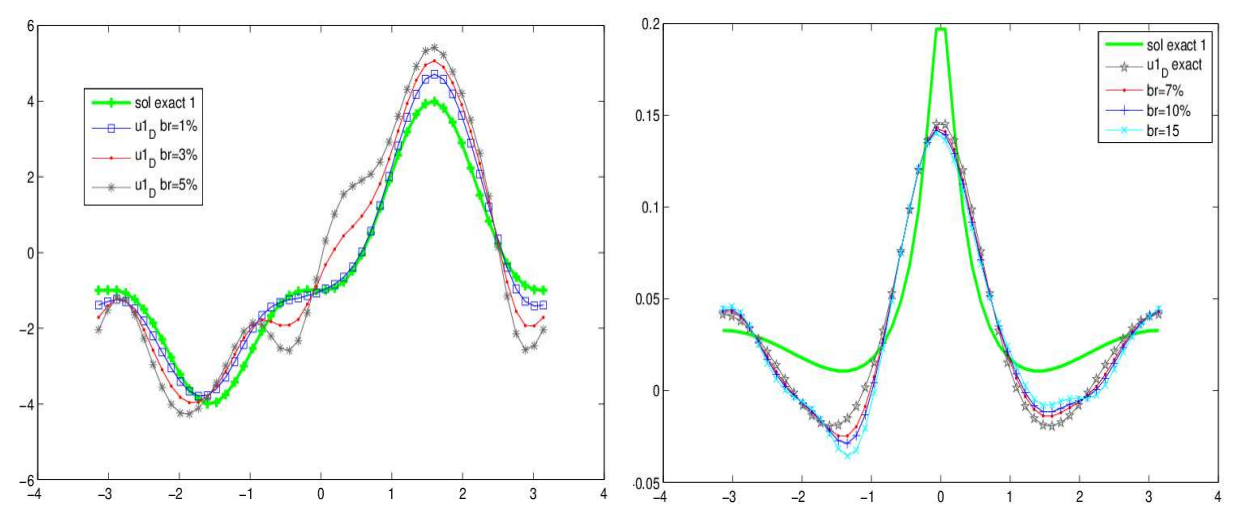

Figure 6. Comparaison of velocity's first component for noisy data : Smooth data(left), Singular data with $a=0.8$ (right)

\section{Application}

As we said in the Introduction, our work was motivated first by the study of airway resistance in pneumology which characterizes the patient's ventilation capability [19] and essentially by the study of the resistivity of the stent which is a medical device used to prevent rupture of aneurysms [14] where the stent is modelized as a porous media with a resistivity $R$.

The problem of identifying Robin coefficient has been studied by Chaabane and Jaoua [11] for Laplace equations and by Boulakia, Egloffe and Grandmont [10] for Stokes problem where they consider the full overdetermined problem namely the velocity and the 
hole stress tensor on $\Gamma_{c}$.

We consider for our aim the following problem :

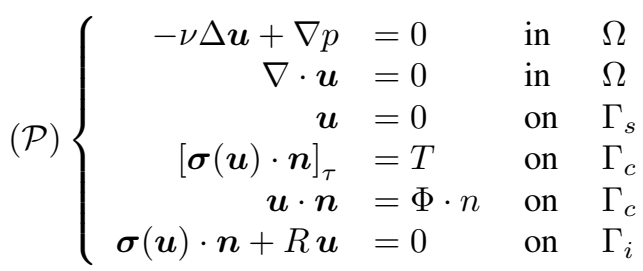

where $R$ is the Robin coefficient assumed hereafter to be a positive number. We want to determine the coefficient $R$ from the knowledge of $\boldsymbol{u} . \tau$ on $\Gamma_{c}$.

We begin by recovering the lacking velocity and normal stress on $\Gamma_{i}$ as explained in the previous sections, then we determine the value of the real parameter $R$ using the formula :

$$
|R|=\left|\frac{\int_{\Gamma_{i}}\left[\boldsymbol{\sigma}\left(\boldsymbol{u}_{2}\right) \cdot n\right]_{1}+\int_{\Gamma_{i}}\left[\boldsymbol{\sigma}\left(\boldsymbol{u}_{2}\right) \cdot \boldsymbol{n}\right]_{2}}{\int_{\Gamma_{i}}\left[\boldsymbol{u}_{2}\right]_{1}+\int_{\Gamma_{i}}\left[\boldsymbol{u}_{2}\right]_{2}}\right|
$$

where for a vector $\boldsymbol{u}$ of $\mathbb{R}^{2},[\boldsymbol{u}]_{k}$ denotes the $k^{t h}$ component of $\boldsymbol{u}$.

We give the numerical results for two different choices of the domain $\Omega$. The first choice corresponds to an annular domain and the second to a rectangular one. For each case and for different test values of $R$, we will compare on $\Gamma_{i}$ the normal stress of $\boldsymbol{u}_{1}$ and $\boldsymbol{u}_{2}$ respective solutions of (13) and (14) with the limit condition $R \boldsymbol{u}_{\text {exact }}$ then we will reconstruct the value of the Robin coefficient that we will call $\rho$ and compare it with the exact used value.

First example : Let $\Omega$ be the annular domain with radius $R_{1}=1$ and $R_{2}=2 . \Gamma_{c}$ will be the outer circle and $\Gamma_{i}$ the inner one (Figure 6). We mesh with 150 nodes on $\Gamma_{c}$ and 100 nodes on $\Gamma_{i} \cdot \varepsilon=6 \times 10^{-4}$ ( 80 iterations were required).

The reconstructed stress tensor on $\Gamma_{i}$ from $\boldsymbol{u}_{1}$ and $\boldsymbol{u}_{2}$ are compared with the one from the exact solution (Figure 7). We give the result for $R=20$ but the numerical tests are done for several values of $R$ and the results are satisfying.

In table 1, we compare the exact value of the Robin coefficient $R$ with the identified one by our method called $\rho$, we note that the error rate is interesting and varies between $0.5 \%$ and $8.9 \%$. 

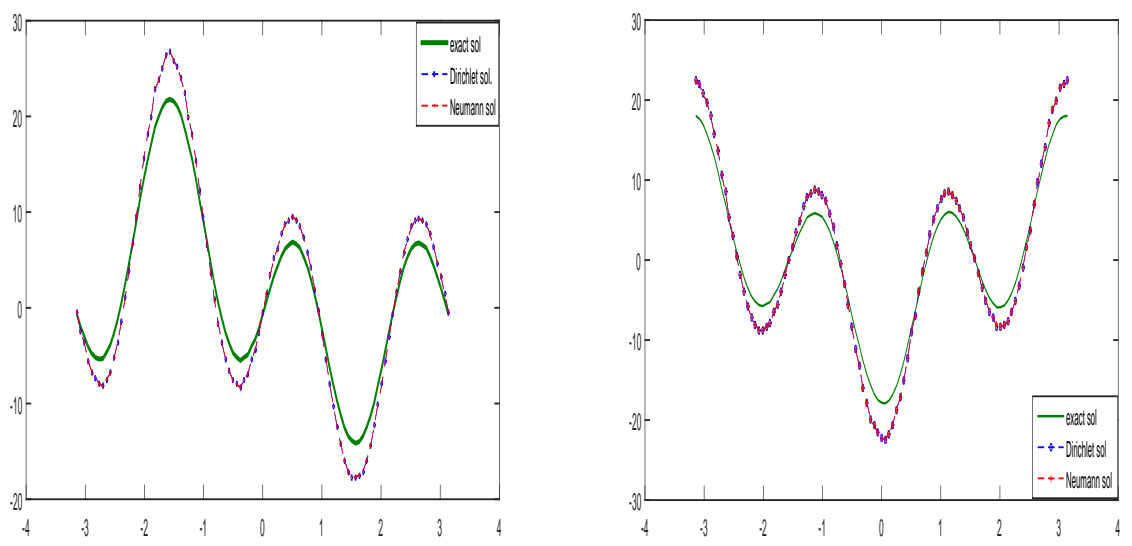

Figure 7. First example with smooth data, $R=20$ : the reconstructed stress tensor on $\Gamma_{i}$

Second example: In this case, $\Omega$ is a rectangular domain with $L=2$ and $\ell=1 . \partial \Omega=$ $\Gamma_{c} \cup \overline{\Gamma_{i} \cup \Gamma_{s}, \text { where }} \Gamma_{c}=[0,2] \times\{1\}, \Gamma_{i}=[0,2] \times\{0\}, \Gamma_{s}=(\{0\} \times[0,1]) \cup(\{2\} \times[0,1])$. We mesh with 60 nodes on $\Gamma_{c}$ and $\Gamma_{i}$, and with 50 nodes on $\Gamma_{s} . \varepsilon=3 \times 10^{-3}$ (50 iterations were required).

In Figure 8 we plot the lacking component of the normal stress on $\Gamma_{c}$ (left) and compare the normal stress with $R \boldsymbol{u}_{\text {exact }}$ on $\Gamma_{i}$ (right). Note that these reconstructed fields are in close agreement with the exact ones. We test for several values of $R$.

In table 2 we reconstruct the value of the Robin coefficient $\rho$ and compare it with the exact one $R$. The error rate is varing between $1.2 \%$ and $7 \%$.

Tableau 1. Comparaison of $\rho$ and $R$ : The annular domain

\begin{tabular}{|c|c|c|c|c|c|}
\hline$R$ & 5 & 10 & 50 & 70 & 100 \\
\hline$\rho$ & 5.07301 & 9.94297 & 45.5175 & 67.1686 & 93.8794 \\
\hline
\end{tabular}



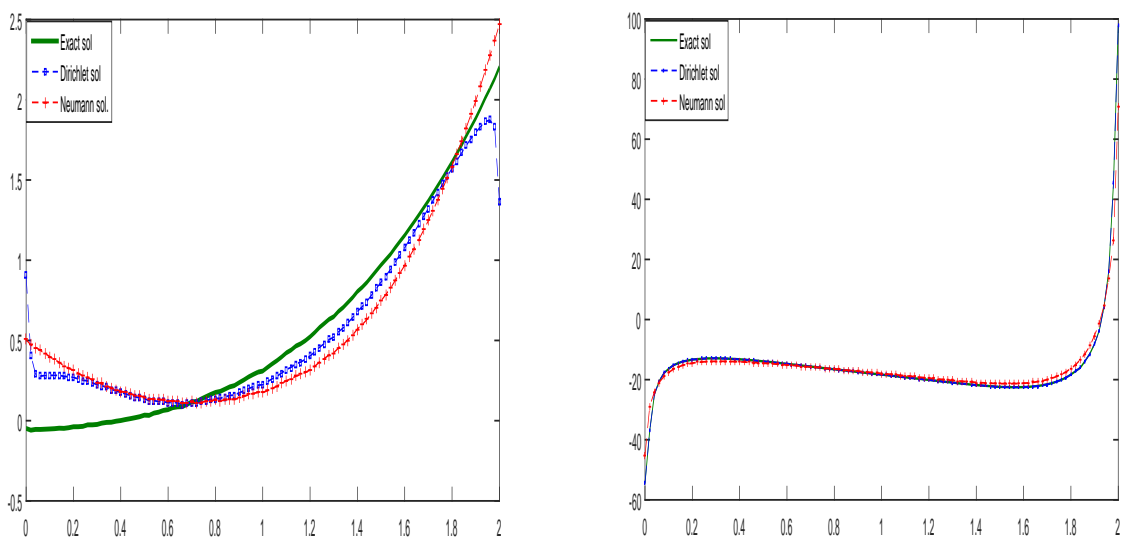

Figure 8. Second example with smooth data, $R=100$ : the reconstructed data on $\Gamma_{c}$ (left) and comparing normal stress with $R u_{\text {exact }}$ on $\Gamma_{i}$ (right)

\section{Conclusion}

In spite of a great amount of work treating of the numerical resolution of Cauchy problems, very few publications are devoted to the Stokes system. Our contribution deals with partially overdetermined boundary data which was not treated up to our knowledge. The purpose here is to treat numerically such an inverse problem. The proposed method seems encouraging, especially for capturing singular data. The forthcoming task is to get stability estimates and to adapt the proposed method for 3D situations.

Tableau 2. Comparaison of $\rho$ and $R$ : The rectangular domain

\begin{tabular}{|c|c|c|c|c|c|c|}
\hline$R$ & 2 & 5 & 10 & 20 & 50 & 100 \\
\hline$\rho$ & 2.05149 & 4.93797 & 9.63617 & 18.8812 & 46.4296 & 92.9558 \\
\hline
\end{tabular}




\section{Bibliographie}

[1] R. Aboulaïch, A. Ben Abda And M. Kallel, «A control type method for solving the Cauchy-Stokes problem », Applied Mathematical Modelling, vol. 37, 2013.

[2] C. Alvarez, C. Conca, L. Friz, O. Kavian and J.H. OrtegA, « Identification of immersed obstacles via boundary measurements », Inverse Problems, vol. 21, 2005.

[3] S. Andrieux, T.N. Baranger And A. Ben AbDa, " Solving Cauchy problems by minimizing an energy-like functional », Inverse problems, vol. 22, 2006.

[4] C. Amrouche And V. Girault, « Decomposition of vector spaces and application to the Stokes problem in arbitrary dimension », Czechoslovak Mathematical Journal, vol. 44, 1994.

[5] C. Amrouche AND N. H. Seloula, « Stokes equations and elliptic systems with nonstandard boundary conditions », Comptes Rendus Mathematiques, vol. 349, 2011.

[6] A. BALleRINI, «Stable determination of an immersed body in a stationary Stokes fluid », Inverse Problems, vol. 26, 2010.

[7] A. Ben AbDa, I. Ben SAad And M. Hassine, « Recovering boundary data : The Cauchy Stokes system », Applied Mathematical Modelling, vol. 37, 2013.

[8] F. Ben Belgacem And H. El FeKIH, « On Cauchy's problem : I. A variational SteklovPoincaré theory », Inverse Problems, vol. 21, 2005.

[9] I. BEN SAAD, «Complétion de données pour le système de Stokes », Ph.D. thesis, ENIT, 2011.

[10] M. Boulakia, A.-C. Egloffe, And C. GRAndmont, « Stability estimates for a robin coefficient in the two-dimensional stokes system », Mathematical Control and Related Field, vol. 3, 2013.

[11] S. ChaAbane, M. JaOUA, «Identification of Robin coefficients by the means of boundary measurements », Inverse Problems, vol. 15, 1999.

[12] K.N. Christodoulou AND L.E. SCRIVEN, « The fluid mechanics of slide coating », Journal of Fluid Mechanics, vol. 208, 1989.

[13] M. M. DENN, « Issues in viscoelastic fluid mechanics », Annual Review of Fluid Mechanics, vol. 22, 1990.

[14] M.A. Fernandez, J.F. Gerbeau, V. Martin, « Numerical simulation of blood flows through a porous interface », ESAIM : Mathematical Modelling and Numerical Analysis, vol. 42, 2008.

[15] A. V. FURSIKOV, « Optimal control of distributed systems. Theory and applications », American Mathematical Soc., 2000.

[16] H. HeCK, X. LI AND J. WANG, « Identification of viscosity in an incompressible fluid », Indiana Univ. Math. J.,vol. 56, 2007.

[17] H. Hedenmalm, « On the uniqueness theorem of Holmgren », Mathematische Zeitschrift, vol. 281, 2015.

[18] B. Maury, « Méthode des Éléments Finis en Élasticité », Ecole Polytechnique, 2007. 
[19] B. MAURY, « The resistance of the respiratory system, from top to bottom », ESAIM : Proceedings and surveys, vol. 47, 2014.

[20] V. MILISIC, «Blood-flow modelling along and trough a braided multi-layer metallic stent», arXiv preprint, vol. arXiv :0908.2513, 2009.

[21] C. Neto, D. R. Evans, E. Bonaccurso, H. J. Butt and V. S. Craig, « Boundary slip in Newtonian liquids : a review of experimental studies », Reports on Progress in Physics, vol. $68,2005$.

[22] D. Peterseim And S.A. Sauter, « Finite element methods for the Stokes problem on complicated domains », Computer Methods in Applied Mechanics and Engineering, vol. 200, 2011.

[23] O. Pironneau, F. Hecht, A. Hyaric and K. Ohtsuka, «FreeFEM », URL : http ://www. freefem. org, 2006.

[24] S. Tateshima, Y. Murayama, J. P. Villablanca, T. Morino, K. Nomura, K. TaNISHITA AND F. VIÑUELA, «In vitro measurement of fluid-induced wall shear stress in unruptured cerebral aneurysms harboring blebs », Stroke, vol. 34, 2003.

[25] R. VERFÜRTH, « Finite element approximation on incompressible Navier-Stokes equations with slip boundary condition », Numerische Mathematik, vol. 50, 1986.

[26] J.J. Wentzel, R. Krams, J. C. Schuurbiers, J. A. Oomen, J. Kloet, W. J. Van DER GIESSEN, P. W. SERRUYS AND C. J. SLAGER, « Relationship between neointimal thickness and shear stress after Wallstent implantation in human coronary arteries », Circulation, vol. 103, 2001. 